\title{
Finasteride monotherapy maintains stable lower urinary tract symptoms in men with benign prostatic hyperplasia following cessation of alpha blockers
}

\author{
J. Curtis Nickel, MD; ${ }^{*}$ Jack Barkin, MD; ${ }^{\dagger}$ Caroline Koch, PhD; ; Charles Dupont, MSc; ${ }^{\xi}$ Mostafa Elhilali, MD;", \\ on behalf of the PROACT investigators
}

\begin{abstract}
Objective: Our Canadian multicentre open-label study sought to evaluate, in patients with moderate/severe lower urinary symptoms (LUTS) secondary to benign prostatic hyperplasia, the effect on symptoms of 9 months of monotherapy with finasteride $5 \mathrm{mg}$ following 9 months of combination treatment (finasteride with an $\alpha$-blocker) as quantified according to the International Prostate Symptom Score (IPSS).
\end{abstract}

Methods: The primary outcome measure for efficacy was the maintenance of IPSS response after cessation of the $\alpha$-blocker. Subjects were treated with a combination of finasteride and an $\alpha$-blocker for 9 months and then with finasteride alone for 3 or 9 months.

Results: Results showed that the IPSS scores after 3 months of monotherapy were within the criteria for equivalence to those after 9 months of combination therapy. Symptom control equivalence was also found after 9 months of monotherapy. The IPSS response rate was also similar for combination and monotherapy. The safety profile was similar and as expected with these medications.

Conclusion: Control of LUTS associated with BPH thus appears to be maintained for at least 9 months with finasteride alone, following a 9-month course of combination therapy with finasteride and an $\alpha$-blocker, with similar safety profiles.

CUAJ 2008;2(1):16-21

\section{Introduction}

For patients with lower urinary tract symptoms (LUTS) associated with benign prostatic hyperplasia $(\mathrm{BPH})$, combination therapy with an $\alpha$-blocker and a 5- $\alpha$ reductase inhibitor (5-ARI) has been shown to be superior to monotherapy with either agent for amelioration of LUTS and prevention of BPH-related disease progression. ${ }^{1}$ Combination therapy has been shown to be tolerated well by most men, although the incidence of adverse events was understandably higher than with either therapy alone. The rationale behind combination therapy is that the 2 therapies have complementary modes of action in managing LUTS secondary to $\mathrm{BPH}$ : the $\alpha$-blocker relaxes the prostatic smooth muscle and increases urinary flow, ${ }^{2}$ and the 5 -ARI reduces the size of the prostate by inhibiting synthesis of dihydrotestosterone. ${ }^{3}$ However, it may not be necessary to continue the $\alpha$-blocker therapy. We performed an open-label multicentre trial to investigate whether symptomatic relief could be maintained with the 5-ARI finasteride alone for up to 9 months, following 9 months of combination therapy using both finasteride and an $\alpha$-blocker.

\section{Methods}

This prospective open-label multicentre Canadian study was designed to evaluate the equivalence of the efficacy and safety of combination therapy (finasteride $5 \mathrm{mg}$ and $\alpha$-blocker) for 9 months followed by monotherapy (finasteride alone) for 3 months (main study). The protocol was amended to allow subjects who completed the initial 12 months of therapy to continue on monotherapy for an additional 6 months, which allowed us to compare 9 months of combination therapy with a subsequent 9 months of finasteride alone (extension study). The primary outcome measure for efficacy was the maintenance of symptom response after cessation of the $\alpha$-blocker.

Eligible patients included men who were at least 45 years old with a diagnosis of $\mathrm{BPH}$ and who were being, or should have been, treated medically for their LUTS. They were also required to have had an International Prostate Symptom Score (IPSS) of more than 12 points before any treatment with an $\alpha$ blocker. If the patient was not presently on a stable dosage of any $\alpha$-blocker, then tamsulosin $0.4 \mathrm{mg}$ daily was provided for 9 months. 
Baseline IPSS was the score before any BPH treatment. Patients with clinically significant concomitant illnesses were excluded. Subjects were evaluated at baseline and months 3, 9, 12 and, for those who elected to remain in the extension study, months 15 and 18.

The primary end point was the change in IPSS from month 9 (combination therapy) to month 12 (monotherapy) for the main study and to month 18 for the extension study. Equivalence criteria were defined as a difference of 2 points or less in the IPSS after 3 and 9 months of monotherapy when compared with the IPSS obtained after 9 months of combination therapy. The hypothesis was tested with a 1-tailed paired test, with $d$ set at 2.0, meaning that a difference of 2.0 or less in the IPSS between month 9 (end of combination therapy) and month 12 or 18 (monotherapy) indicated equivalence of combination therapy and monotherapy. Secondary end points included the quality of life component of the IPSS questionnaire, prostate specific antigen (PSA) level, patients' and physicians' assessments of symptom control and safety.

Given the methodological design of the study, statistical analyses were performed with per protocol subjects set as suggested by International Conference on Harmonisation $(\mathrm{ICH})$ guidelines (topic E9). Paired and unpaired analyses of variance were performed for continuous outcomes; nominal outcomes were analyzed with chisquare and McNemar tests. Fisher's protected least significant difference tests were used for post hoc pair-wise comparisons. All results are reported for the main study (up to month 12, i.e., 9 months of combination therapy followed by 3 months of monotherapy) and the extension study (up to month 18, i.e., 9 months of combination therapy followed by 9 months of monotherapy). Statistical analyses were performed with SYSTAT 11.0 (Systat, Cranes Software International, 2004); a $p$ value $<0.05$ was considered statistically significant.

\section{Results}

A total of 275 men with a mean age of 66.1 (standard deviation [SD] 7.7) years agreed to participate in the main study and signed an informed consent. Recruitment ran from April 2004 to May 2005, and the last subject was seen in December
2006. Major recruiting provinces were Ontario (55.3\%), Quebec (19.6\%) and British Columbia (16.4\%). Of the 275 subjects initially enrolled, 55 discontinued during the combination phase (first 9 months). Of those, 31 (56\%) discontinued for an adverse event (AE). Of the 220 subjects who started monotherapy, 47 discontinued before month 12 . Of those, 26 (55\%) discontinued for an AE. Thus 173 subjects completed the main study per-protocol; $124(72 \%)$ agreed to participate in the extension study, thus maintaining monotherapy to month 18 . Of those, 17 subjects discontinued before month 18, with 5 (29\%) discontinuing for an AE. Thus 107 subjects completed extension study per protocol. Primary baseline characteristics of the total patient population are outlined in Table 1.

Primary end point analysis of the main study

Table 1: Baseline characteristics of the total patient population

\begin{tabular}{|c|c|c|}
\hline \multirow[b]{2}{*}{ Characteristic } & \multicolumn{2}{|c|}{$\%$ of patients* } \\
\hline & $\begin{array}{l}\text { Main study; } \\
\quad n=275\end{array}$ & $\begin{array}{l}\text { Extension study; } \\
\qquad n=124\end{array}$ \\
\hline Mean age, yr (and SD) & $66.1(7.7)$ & $66.2(8.2)$ \\
\hline White & 92.4 & 93.6 \\
\hline \multicolumn{3}{|l|}{ Prostate enlarged } \\
\hline Mildly (< $30 \mathrm{~g})$ & 1.8 & 1.6 \\
\hline Moderately (30-40 g) & 66.4 & 66.9 \\
\hline Severely (> $40 \mathrm{~g}$ ) & 31.8 & 31.5 \\
\hline $\begin{array}{l}\text { Mean TRUS prostate } \\
\text { volume (mL), no (and SD) }\end{array}$ & $65.1(28.6)$ & $69.2(27.5)$ \\
\hline $\begin{array}{l}\text { Mean time since first } \\
\text { diagnosis of BPH, yr } \\
\text { (and SD) }\end{array}$ & $2.8(3.8)$ & $2.8(3.8)$ \\
\hline Naive to $\alpha$-blockers & 63.3 & 62.9 \\
\hline \multicolumn{3}{|l|}{ On $\alpha$-blocker } \\
\hline Tamsulosin & 63 & 72 \\
\hline Terazosin & 16 & 13 \\
\hline Alfuzosin & 14 & 9 \\
\hline Doxazosin & 7 & 6 \\
\hline \multicolumn{3}{|l|}{ Time on $\alpha$-blocker } \\
\hline$<3 \mathrm{mo}$ & 22.8 & 28.3 \\
\hline $3 \mathrm{mo}-1 \mathrm{yr}$ & 27.7 & 23.9 \\
\hline$>1 \mathrm{yr}$ & 49.5 & 47.8 \\
\hline \multicolumn{3}{|l|}{ Medical history } \\
\hline Hypertension & 40.0 & 43.5 \\
\hline Diabetes & 14.2 & 16.9 \\
\hline
\end{tabular}


showed a mean IPSS difference of 1.26 (SD 5.38) (95\% confidence interval [Cl] 0.58-1.94; $p=0.04$ ), confirming the efficacy equivalence hypothesis. There was a statistically significant increase in IPSS from month 9 (mean 11.97, SD 5.98) to month 12 (mean 13.23, SD 6.44), but the mean difference and its $\mathrm{Cl}$ still fell below the preset equivalence criteria of 2.0. Primary end point analysis of the extension study confirmed this result (mean IPSS difference of -0.44 , SD 4.70$)(95 \% \mathrm{Cl}-1.15$ to $0.27 ; p<0.001))$. At the end of the extension study, $79.8 \%$ of subjects met the equivalence criteria of 2.0 or less point change on the IPSS (month 18 compared with month 9).

Secondary outcome measures are detailed in Table 2. During the main study, the quality of life component of the IPSS improved (the score decreased) from baseline to month 9 and then slightly deteriorated from month 9 to month 12 , with statistical significance. Urination problems showed a similar pattern of improvement from baseline to month 9 followed by a slight deterioration at month 12 . Voiding symptoms improved from baseline to month 9, and the improvement was maintained at month 12 (difference not statistically significant). PSA levels significantly decreased by about $43 \%$ from baseline to month 9 and then remained stable from month 9 to month 18. For the extension study, post hoc Fisher's tests showed no statistically significant changes from the end of combination therapy (month 9) to the end of monotherapy (month 18) for the IPSS quality of life component, urination problems, voiding symptoms assessment and PSA levels.
The continuous IPSS end point was also recoded as dichotomous (responder $=$ decrease $\geq 3$ IPSS points from baseline). At month 9, the end of the combination therapy phase, $80.3 \%$ of patients were classified as responders; at month 12 , 3 months into the monotherapy phase, $74.0 \%$ were classified as responders ( $v$. baseline). This decrease was not statistically significant (month $9 \mathrm{v}$. month $12, p=0.09$ ). At month 18, after 9 months of monotherapy, the response rate was $80.5 \%$ ( $p=$ 0.69 v. month 9).

Safety results showed a total of 324 nonserious adverse events (NSAEs) occurring during the main study in 142 subjects (mean 2.3, SD 1.6, NSAEs per subject with at least 1 NSAE or 1.2, SD 1.6, in all 275 subjects), an incidence that is consistent with past clinical trials and product monograms of both study medications. Of all NSAEs, $66.1 \%$ started during the combination therapy; $28.8 \%$ of all NSAEs led to discontinuation of therapy. Of all NSAEs, $32.7 \%$ were considered possibly or probably or definitely related to study medication(s). Of all NSAEs, $37.2 \%$ were urinary or sexual. The most frequent NSAEs reported during the main study (first 12 months) were decreased libido $(5.2 \%)$, impaired urination $(5.2 \%)$, urinary frequency $(4.6 \%)$, decreased erection $(3.7 \%)$, dizziness $(3.1 \%)$ and decreased ejaculation $(2.8 \%)$.

In the extension study, a total of 142 NSAEs occurred in 57 subjects (46.0\%) who experienced at least 1 NSAE (mean 2.5, SD 1.7, NSAEs per subject). Of the 142 NSAEs, 91 (64.1\%) occurred during the combination therapy, $29 \%$ were urinary or

Table 2: Endpoint results for main and extension studies

\begin{tabular}{|c|c|c|c|c|c|c|c|}
\hline \multirow[b]{2}{*}{ Measure } & \multicolumn{7}{|c|}{ Mean score (and SD)* } \\
\hline & Baseline & Mo 9 & $\begin{array}{l}p \text { value v. } \\
\text { baseline }\end{array}$ & Mo 12 & $\begin{array}{c}p \text { value } \mathrm{v} . \\
\text { mo } 9\end{array}$ & Mo 18 & $\begin{array}{c}p \text { value } \mathrm{v} . \\
\text { mo } 9\end{array}$ \\
\hline IPSS & $19.93(5.11)$ & $11.97(5.98)$ & $<0.001$ & $13.23(6.44)$ & 0.01 & $11.59(6.69)$ & 0.41 \\
\hline QoL-IPSSt & $3.82(1.23)$ & $2.38(1.27)$ & $<0.001$ & $2.62(1.36)$ & 0.01 & $2.21(1.38)$ & 0.11 \\
\hline PSA, ng/mL & $3.48(2.16)$ & $2.00(1.41)$ & $<0.001$ & $1.97(1.45)$ & 0.70 & $1.94(1.61)$ & 0.92 \\
\hline $\begin{array}{l}\text { Urination } \\
\text { problem }\end{array}$ & $3.50(1.15)$ & $4.74(1.12)$ & $<0.001$ & $4.39(1.12)$ & 0.01 & $4.71(1.22)$ & 0.94 \\
\hline $\begin{array}{l}\text { Voiding } \\
\text { symptoms§ }\end{array}$ & $2.48(0.83)$ & $2.98(0.78)$ & $<0.001$ & $2.84(0.79)$ & 0.06 & $3.02(0.83)$ & 0.52 \\
\hline \multicolumn{8}{|c|}{$\begin{array}{l}\text { SD = standard deviation; IPSS = international prostate symptom score; QoL = quality of life; PSA = prostate specific antigen. } \\
\text { *Unless otherwise indicated. } \\
\text { tQuality of life due to urinary symptoms was scored on a } 0 \text { to } 6 \text { scale, } 6 \text { indicating a terrible quality of life. } \\
\text { fUrination problems were scored on a } 1 \text { to } 7 \text { scale ( } 1 \text { indicating the most severe symptom and } 7 \text { indicating no problems). } \\
\text { §Voiding symptoms assessment reported by patients was scored on a scale of } 1 \text { to } 4 \text { (1 indicating voiding symptoms not controlled at all and } 4 \text { indicating voiding symptoms } \\
\text { well controlled). }\end{array}$} \\
\hline
\end{tabular}


sexual and $22 \%$ were considered possibly or probably or definitely related to study medication(s). Table 3 highlights serious adverse experience (SAE) information for the main and extension studies.

\section{Discussion}

$\mathrm{BPH}$ is a common condition in aging men, and about one-half of men who have a BPH diagnosis have moderate-to-severe LUTS affecting quality of life and requiring treatment. LUTS associated with $\mathrm{BPH}$ can be managed medically with either $\alpha$-blockers, 5-ARIs, or a combination of both. Monotherapy with either $\alpha$-blockers or 5 -ARIs is effective in reducing symptoms of LUTS; however, $\alpha$-blockers provide more rapid symptomatic relief. Only 5-ARIs reduce progression of symptoms, the long-term risks of acute urinary retention and the need for invasive surgery. ${ }^{4,5}$

Combination therapy can offer the rapid relief of symptoms associated with $\alpha$-blockers and the long-term benefits of 5-ARIs. Combination therapy is well tolerated, with the risk of side effects being almost equivalent to the sum of the side effects of the individual therapies. ' Several studies have demonstrated that the $\alpha$-blocker can be withdrawn after an interval of combination therapy, after which symptom control is maintained with a 5-ARI alone. ${ }^{6-8}$ The optimal duration of combination therapy seems to be around 9 months because $84 \%$ of the men were able to discontinue $\alpha$-blockers after 9 months with no increase in symptoms.' However, the follow-up interval of 5-ARI monotherapy lasted only 1 month.
This study evaluated whether monotherapy with the 5-ARI finasteride alone, after 9 months of combination therapy of finasteride plus an $\alpha$-blocker, could maintain symptomatic control of LUTS secondary to BPH for a 3- or 9-month interval. Three months of monotherapy were evaluated in 173 patients in the main study, and 9 months of monotherapy were evaluated in 124 patients in the extension study. Both courses of monotherapy were found to be equivalent to combination therapy in the control of LUTS, as evaluated by the IPSS scores. Equivalence was defined in this study as a mean IPSS difference of 2.0 or less between the end of the combination therapy phase and the end of a monotherapy phase: the month 9 IPSS scores versus the month 12 scores gave a mean IPSS difference of 1.26 (SD 5.38) $(95 \% \mathrm{Cl}$ $0.58-1.94 ; p=0.036$ ), and the month 9 scores versus the month 18 scores gave a mean IPSS difference of -0.44 (SD 4.70) (95\% Cl-1.15 to 0.27; $p<0.001)$. The rationale for choosing this parameter of 2.0 or less is based on published data suggesting that patients will only perceive an improvement in symptom score of 3 or more. ${ }^{9}$

The secondary outcome measures showed that quality of life scores and urination problems both improved from baseline to month 9 and showed no statistically significant changes between month 9 and month 18. PSA levels and voiding symptoms decreased from baseline to month 9 , and these measures also showed no statistically significant changes between month 9 and month 18. This indicates that 9 months of monotherapy can maintain the improvement obtained during

\begin{tabular}{|c|c|c|c|c|}
\hline \multirow[b]{3}{*}{ Type of SAE } & \multicolumn{4}{|c|}{ Group; no. of SAEs* } \\
\hline & \multicolumn{2}{|c|}{ Main study; $† n=275$} & \multicolumn{2}{|c|}{ Extension study; $¥ n=124$} \\
\hline & $\begin{array}{l}\text { Monotherapy } \\
\text { (mo 9-12) }\end{array}$ & $\begin{array}{c}\text { Combination } \\
\text { therapy } \\
\text { (mo 0-9) }\end{array}$ & $\begin{array}{l}\text { Monotherapy } \\
\text { (mo 9-18) }\end{array}$ & $\begin{array}{c}\text { Combination } \\
\text { therapy } \\
\text { (mo 0-9) }\end{array}$ \\
\hline No. of SAEs§ (and \%) & $18(72)$ & $7(28)$ & $4(57)$ & $3(43)$ \\
\hline $\begin{array}{l}\text { No. of SAEs related to } \\
\text { study medication(s) }\end{array}$ & 3 & 0 & 19 & 0 \\
\hline $\begin{array}{l}\text { No. of SAEs leading to } \\
\text { study discontinuation }\end{array}$ & 3 & 0 & 1 & 0 \\
\hline $\begin{array}{l}\text { SAE = serious adverse event. } \\
\text { *Unless otherwise indicated. } \\
\text { +Total SAEs } 25 \text {. } \\
\text { \#Total SAEs } 7 \text {. } \\
\text { SIncludes } 4 \text { deaths: none relate } \\
\text { qDizziness possibly related to } s\end{array}$ & $\begin{array}{l}\text { dication(s). } \\
\text { on(s) which }\end{array}$ & 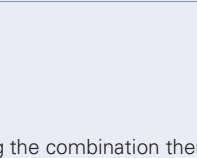 & & \\
\hline
\end{tabular}


Nickel et al

the combination phase. For the quality of life and urination problem measures, there was a small but statistically significant deterioration from month 9 to month 12 . However, these same measures showed no changes between month 9 and month 18.

To better understand the study results and limitations, it is important to describe the typical patient profile of those who completed the main study and those who completed the extension study. Patients who did experience a worsening of some symptoms during the first 3 months of monotherapy might have elected not to continue the study into the extension phase. A comparison of patients who continued with the 6-month monotherapy extension and those who were eligible but declined participation reveals similar IPSS at baseline $(p=0.06)$ and at the end of the combination therapy phase $(p=0.81)$; however, after 3 months of monotherapy, those who elected not to participate had a significant increase in their IPSS $(p<0.001)$. This would suggest that, for the patients able to maintain symptom control on finasteride alone after 9 months of combination therapy, this stable control of symptoms can be maintained for at least 9 months.

The potential limitations of the current study are related to the open-label and single-cohort design. However, the open-label design is necessary because blinding with respect to the treatment is not compatible with emulation of the reallife setting. The strengths of the current study include the random selection of physicianinvestigators and patients from the Canadian population, which permits generalization of the results to the target population. The use of standardized, self-administered questionnaires reduced potential ascertainment bias.

In our study, as in previous studies, combination therapy and monotherapy with finasteride were both well tolerated.1,10,11 The most frequent NSAEs reported in the main study were decreased libido, impaired urination, urinary frequency, decreased erectile function, dizziness and decreased ejaculation.

In summary, we have shown that control of LUTS associated with BPH can be maintained for at least 9 months with the use of finasteride alone, following a 9-month course of combination therapy with finasteride and an $\alpha$-blocker. Maintenance of symptomatic control using finas- teride alone not only reduces the cost and inconvenience of a second medication but will also diminish the risk of side effects, which is increased by taking 2 types of drugs.

From the *Department of Urology, Queen's University, Kingston General Hospital, Kingston, Ont., †Chief of Staff, Humber River Regional Hospital, Director: The Male Health Centre, North York, Director: Can-Am HIFU, Assistant Professor, Department of Surgery, University of Toronto, Toronto, Ont., ‡Medical Programs Manager, Merck Frosst Canada Ltd., Pointe-Claire-Dorval, Que., §Research Consultant, DataMed Inc., St-Jérôme, Que., TChairman of the Department of Surgery, and Stephen Jarislowsky Chair in Urology at McGill University and Surgeon-in-Chief, Royal Victoria and Montreal General Hospitals, Montréal, Que.

Acknowledgements: The authors thank the following investigators for their contribution. British Columbia: A. Charalambos, L. Goldenberg, D. Lavoie, K. Prestage, P.J. Pommerville. Saskatchewan: P. Lau. Manitoba: J. Milner. Ontario: J. Barkin, R. Casey, K. Jansz, L. Klotz, W-S Leung, W. Love Jr., A. Mathur, C. Nickel, H. Razvi, P. Roney, K. Sachdeva, A.M. Skehan, A. Toguri, A.L. Winter. Quebec: L. Aaron, S. Carrier; M. Elhilali, I. Kuzmarov, B. Laroche, A. Maillette, J. Simard. New Brunswick: S. Bryniak. Nova Scotia: W. T'ien.

The trial was funded by a research grantin-aid from Merck Frosst Canada.

This article has been peer reviewed.

Competing interests: Dr. Koch is an employee in Medical Research at Merck Frosst Canada Ltd; Dr. Dupont is the president of DataMed Inc. (CRO); Dr. Nickel, Dr. J. Barkin and Dr. M. Elhilali are consultants for the PROACT study. Dr. Nickel has been a member of an Advisory Board for Merck Frosst, GlaxoSmithKline, Boston Scientific, Farr Laboratories and Triton Pharma. He has been involved in scientific trials with Merck Frosst, GlaxoSmithKline, Sanofi Aventis, Allergan and Plethora Solutions. He has been a CME meeting participant with Merck Frosst and Sanofi-aventis. Dr. Barkin has received honoraria for CME and attending Advisory Board meetings for Merck Frosst. Dr. Elhilali has received honoraria for CME and attending Advisory Board meetings for Merck Frosst. All investigators received grants related to the conduct of the study including but not limited to patient recruitment and Case Report Form completion on a prorated basis.

\section{References}

1. McConnell JD, Roehrborn CG, Bautista OM, et al. The long-term effect of doxazosin, finasteride, and combination therapy on the clinical progression of benign prostatic hyperplasia. N Engl J Med 2003;349:2387-98.

2. Andersson KE. Storage and voiding symptoms: pathophysiologic aspects. Urology 2003;62(Suppl 2):3-10.

3. Marks LS, Partin AW, Dorey FJ, et al. Long-term effects of finasteride on prostate tissue composition. Urology 1999;53:574-80.

4. Roehrborn CG. 5-alpha-Reductase inhibitors prevent the progression of benign prostatic hyperplasia. Rev Urol 2003;5(Suppl 5):S12-21.

5. Madersbacher $S$, Marszalek M, Lackner J, et al. The long-term outcome of medical therapy for BPH. Eur Urol 2007;51:1522-33.

6. Baldwin $\mathrm{KC}$, Ginsberg PC, Harkaway RC. Discontinuation of alpha-blockade after initial treatment with finasteride and doxazosin for bladder outlet obstruction. Urol Int 2001;66:84-8.

7. Baldwin $\mathrm{KC}$, Ginsberg $\mathrm{PC}$, oehrborn $\mathrm{CG}$, et al. Discontinuation of alpha-blockade after initial treatment with finasteride and doxazosin in men with lower urinary tract 
symptoms and clinical evidence of benign prostatic hyperplasia. Urology 2001;58:203-9.

8. Barkin J, Guimaraes $M$, Joacobi $G$, et al. Alpha-blocker therapy can be withdrawn in the majority of men following initial combination therapy with the dual 5alpha-reductase inhibitor dutasteride. Eur Urol 2003;44:461-6.

9. Barry MJ, Williford WO, Chang Y, et al. Benign prostatic hyperplasia specific health status measures in clinical research: how much change in the American Urological Association symptom index and the benign prostatic hyperplasia impact index is perceptible to patients? J Urol 1995; 154:1770-4

10. Kirby RS, Roehrborn C, Boyle P, et al. Efficacy and tolerability of doxazosin and finasteride, alone or in combination, in treatment of symptomatic benign prostatic hyperpla- sia: the Prospective European Doxazosin and Combination Therapy (PREDICT) trial. Urology 2003;61:119-26.

11. Lepor H, Williford WO, Barry MJ, et al. The efficacy of terazosin, finasteride, or both in benign prostatic hyperplasia. Veterans Affairs Cooperative Studies Benign Prostatic Hyperplasia Study Group. N Engl J Med 1996;335:533-9.

Correspondence: Dr. J. Curtis Nickel, Department of Urology, Queen's University, Kingston General Hospital, 76 Stuart St., Empire 4, Kingston ON K7L 2V7; jin@queensu.ca

\title{
We welcome your comments on the journal and on specific articles.
}

\author{
All letters will be considered \\ for publication in the journal.
}

\section{Send your letters to the Editor-in-Chief at journal@cua.org}

CUAJ JAUC

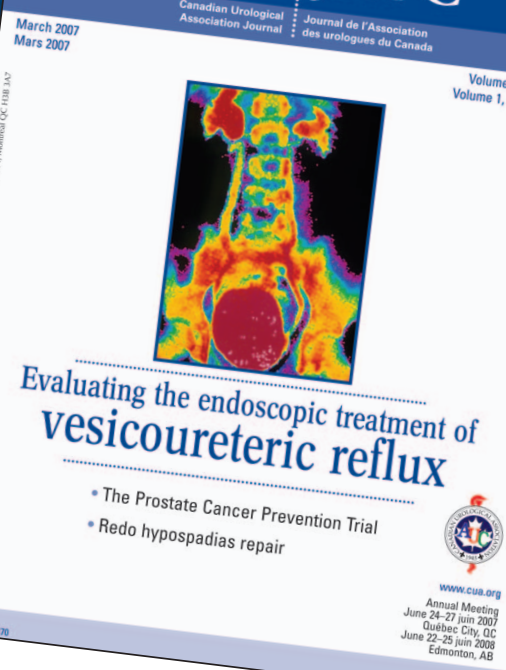

